\title{
Identification of the critical load for cyclic loading of short- fibre reinforced PA6 GF30 by a quantitative analysis of the thermoelastic response
}

\author{
A. Klavzar ${ }^{1, a}$, R. Renz ${ }^{2}$ \\ ${ }^{1}$ ISL French-German Research Institute of Saint-Louis, 68301 Saint-Louis Cedex, France \\ ${ }^{2}$ Technical University of Kaiserslautern, Institute of Resource Centred Product Development (RPE), \\ Kaiserslautern, Germany
}

\begin{abstract}
In TSA the thermoelastic temperature change in the surface of cyclically loaded materials is measured, which is for isotropic materials proportional to the change of the sum of the principal stresses. The sensitivity of the infrared sensors used in TSA is related non-linearly with the absolute temperature. This can be effectively corrected by a direct calibration of the thermal signal, if this is not done by the supplier of the measurement system. The injection moulded PA6 GF 30 has layer-wise changing mechanical and thermoelastic properties. Therefore is a strain oriented approach used to calibrate the thermoelastic signal, the measured thermoelastic signal can be directly related to the sum of the principal strains. In a load increase test the thermoelastic signal increases proportional to the applied load up to a certain load-level. Beyond this load, the thermoelastic signal $\mathrm{S}$ increases disproportionately high compared to the applied load. The increase of the thermoelastic signal is caused by the beginning disruption of the material, what is confirmed by measuring the acoustic emission. Single-load fatigue measurements are done, indicating that the maximum applicable load under cyclic loading conditions could be determined from the thermoelastic signal.
\end{abstract}

\section{Optical measurement of the thermoelastic effect}

In thermoelastic stress analysis (TSA) small temperature changes in solid bodies caused by cyclic loading are measured. For isotropic materials is the thermoelastic temperature change $\Delta \mathrm{T}$ proportional to the change of the sum of the principal stresses in the surface of the material under investigation [1]:

$$
\Delta T=\frac{\alpha}{\rho c_{p}} T_{0} \Delta\left(\sigma_{1}+\sigma_{2}\right)
$$

\footnotetext{
a e-mail : AndreasKlavzar@gmx.de
} 
with the coefficient of thermal expansion $\alpha$, the density $\rho$ and the specific heat capacity $c_{p}$. The absolute temperature of the material under investigation is $\mathrm{T}_{0}$ and $\Delta\left(\sigma_{1}+\sigma_{2}\right)$ is the change of the sum of the principal stresses ( $1^{\text {st }}$ invariant of the stress tensor) due to cyclic loading.

The thermoelastic temperature change $\Delta \mathrm{T}$ is measured optically by measuring the absolute temperature with an infrared sensor with a high framing rate. The thermoelastic temperature change is determined from the change of the thermal signal of the measuring system. The raw, uncalibrated absolute temperature signal $\mathrm{S}_{\mathrm{DC}}$ of the infrared sensors used in TSA is related non-linearly with the absolute temperature. The measured thermoelastic signal $\mathrm{S}$ is developed from this thermal signal $\mathrm{S}_{\mathrm{DC}}$, thus the thermoelastic signal $\mathrm{S}_{\mathrm{DC}}$ changes also non-linearly with the temperature. It was shown by Dulieu-Barton et al. [2], that the signal to temperature relation of the thermal signal $\mathrm{S}_{\mathrm{DC}}$ and the thermoelastic signal $\mathrm{S}$ is following a power law. This non-linear influence of the absolute temperature is not for all measuring systems corrected by the supplier of the system. Users of the DeltaTherm TSA-system for example, which was used in this work, have to correct the measured signals by themselves if severe changes of the absolute temperature are expected in a measurement. This is the case for short-fibre reinforced thermoplastics as they tend to heat under cyclic loading due to their viscoelastic properties.

The influence of the absolute temperature on the thermoelastic signal S can be corrected either by the application of a correction routine directly on the measured thermoelastic signal S [2]. Another method, used in this work, is to calibrate the thermal signal $\mathrm{S}_{\mathrm{DC}}$ against the temperature values [3] by measuring the absolute temperature signal $\mathrm{S}_{\mathrm{DC}}$ at different absolute temperature values and implementing the measured values in a calibration file of the DeltaVision software. After a measurement, a calibration routine is applied to the measured data which calibrates the measured values of the thermal signal $\mathrm{S}_{\mathrm{DC}}$ and the thermoelastic signal $\mathrm{S}$ against temperature values. The calibrated thermoelastic signal $\mathrm{S}$ is proportional to the thermoelastic temperature change given by equation $1, \mathrm{~S} \propto \Delta \mathrm{T}$, with a linear increase with the absolute temperature.

\section{Calibration of the thermoelastic signal of short-fibre reinforced thermoplastics}

\subsection{Mechanical and thermoelastic properties of PA6 GF30}

The short fibre reinforced thermoplastic PA6 GF30 is produced by injection moulding. The injection moulding process leads to a layer-wise changing fibre orientation (FO) in the composite material, resulting in layer-wise changing mechanical and thermoelastic properties. The layers of PA6 GF30 produced by injection moulding are depicted in Figure 1 [4].

The different layers have different mechanical and thermoelastic properties, due to the different fibre orientations. The global mechanical properties of the composite material are dominated by the layers with high fibre orientation 3 and 5, resulting in orthotropic mechanical properties of the composite material [5].

To evaluate the mechanical properties of PA6 GF30 test specimens with two different fibre orientations were prepared from injection moulded plates, see Figure 1. One set of specimens was prepared with main fibre orientation (flow direction) in direction of the specimens' length axes, fibre orientation L (first index 1 of the specimen numbering). Another set had a main fibre orientation perpendicular to the specimens' length axes, fibre orientation $\mathrm{T}$ (first index 2 of the specimen numbering). 


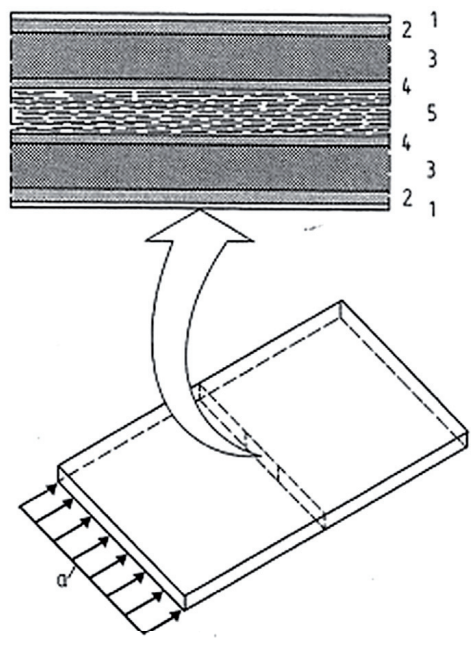

1: Surface layer with low fibre content

2: Layer with random fibre orientation

3: Layer with fibre orientation in flow direction

4: Layer with random fibre orientation

5: Layer with fibre orientation perpendicular to flow direction

a: Flow direction of molten PA6 GF30
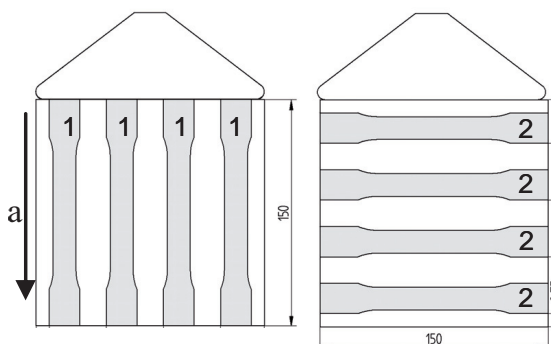

Fibre orientation $\mathrm{L}$ Fibre orientation $\mathrm{T}$

Fig. 1. Fibre orientation in cross section of injection moulded PA6 GF30 [4]

The mechanical properties were measured under quasi-static load conditions with the digital image correlation method (DIC) [6]. The DIC was used as it is an optical strain measurement technique, e.g. the mechanical properties of the specimens are not influenced as it was the case using for example strain gauges [7]. On the other hand is the stiffness of viscoelastic materials under quasistatic conditions lower than under dynamic loading, see the values measured in [5]. In the following only the Poissons' ratios are needed, which are nearly identical for quasi-static and dynamic loading.

The orthotropic mechanical properties measured with the DIC are given in table 1, they were evaluated according to ISO 527-1, e.g. as the secant modulus between 0.05 and $0.25 \%$ strain.

Table 1. Orthotropic mechanical properties of PA6 GF30

\begin{tabular}{|c|c|c|c|}
\hline$E_{1}[\mathrm{MPa}]$ & $\mathrm{E}_{2}[\mathrm{MPa}]$ & $v_{12}$ & $v_{21}$ \\
\hline 5700 & 3440 & 0,45 & 0,26 \\
\hline
\end{tabular}

\subsection{Calibration of the thermoelastic signal of PA6 GF30}

The thermoelastic signal is induced in the isotropic surface layer of the material, on conditions that the loading frequency is high enough to ensure quasi-adiabatic conditions for the temperature change and that an isotropic surface layer of sufficient thickness exists [8].

A strain oriented approach is used to calibrate the thermoelastic signal of a composite material with isotropic thermoelastic properties as PA6 GF30. In the strain oriented approach, the measured thermoelastic signal is not calibrated against the sum of the principal stresses in the surface layer of the material, but against the sum of the principal strains, according to equation $2[5,8]$ :

$$
\Delta T=\frac{\alpha}{\rho c_{p}} \frac{E}{1-v} T_{0} \Delta\left(\varepsilon_{1}+\varepsilon_{2}\right)
$$

The properties E, $v, \alpha, \rho$ and $c_{p}$ are those of the surface layer of the composite material, which consists of the pure matrix material. In the strain oriented approach is the measured thermoelastic 
signal directly related to the change of the sum of the principal strains, measured with a reference measuring system. The stiffness of the different layers and the overall mechanical properties of the composite material have not to be determined as it was the case using a stress oriented approach.

A laserextensometer (LEX) was used in this work as the reference measuring system for the strain. A LEX is a non-contacting optical measuring system, see Figure 2. a, which measures the mechanic-dynamic properties of polymers at forced vibrations [9]. A stress-strain graph of viscoelastic materials under cyclic load forms a hysteresis loop with the characteristic quantities: stresses, strains, stiffness and mechanical energies. During dynamic loading, the material absorbs energy, which is not completely recovered during unloading. This so called energy loss corresponds to the area enclosed by the hysteresis loop (Figure 2. b). The reversibly stored strain energy is fully recovered during unloading.

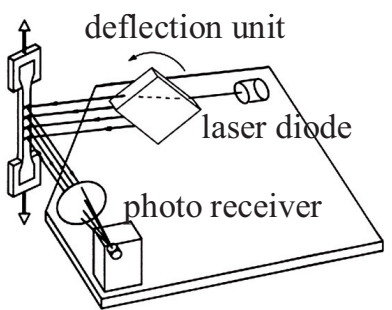

a: laserextensometer

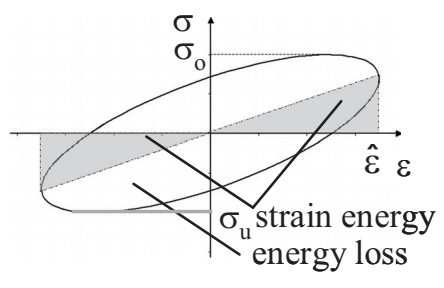

b: stress-strain hysteresis

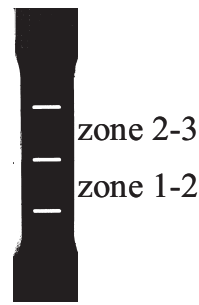

c: specimen with marks

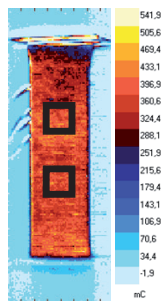

d: TSA-signal

Fig. 2. a: Laserextensometer, b: stress-strain hysteresis, c: test specimen, d: TSA-signal

To measure the strain with the LEX, the surface of the specimen is coloured with white marks, see Figure 2. c. The current distance between two marks is recorded from the intensity of a laser beam reflected from the surface of the cyclically loaded specimen. The specimens in this work were divided by the marks in 2 zones, so the strain component in load direction was measured in 2 areas of the specimen. The strain perpendicular to the load direction was calculated from the measured longitudinal strain component and the Poisson's ratio measured with the DIC, see Table 1.

The TSA signal S of the cyclically loaded specimens was measured simultaneous to the stressstrain hysteresis and evaluated in the areas between the marks for the LEX, see Figure 2. d.

The calibration measurement was done in a load increase test with 6 load steps and a loading frequency of $5 \mathrm{~Hz}$, see the left graph of Figure 3.
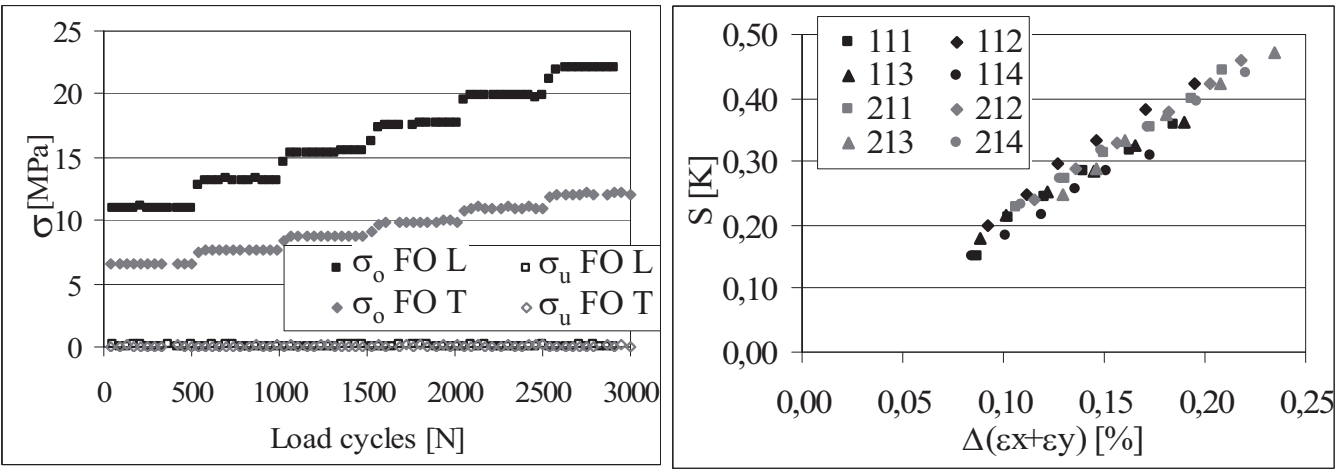

Fig. 3. Thermal signal $\mathrm{S}$ of an aluminium alloy with temperature

One load step had a duration of 500 load cycles, the TSA measurement was performed from the $300^{\text {th }}$ to the $400^{\text {th }}$ load cycle. The upper stresses $\sigma_{o}$ for fibre orientation L ranged from $11 \mathrm{MPa}$ to 22 
$\mathrm{MPa}$. For fibre orientation T the upper stresses were from 6.5 MPa to $12 \mathrm{MPa}$. The lower stress $\sigma_{\mathrm{u}}$ in a load cycle was always $0 \mathrm{MPa}$. The relation between the measured thermoelastic signal $\mathrm{S}$ and the sum of the principal strains is given in the right graph of Figure 3.

The measured thermoelastic signal of PA6 GF30 is proportional to the sum of the principal strains, and nearly independent from the main fibre orientation of the composite material. The factor of proportionality is $0.50 \pm 0.04 \% / \mathrm{K}$ for fibre orientation $\mathrm{L}$ and $0.48 \pm 0.01$ for fibre orientation $\mathrm{T}$.

In [5] the calibration constant of PA6 GF 30 was evaluated with $0.41 \% / \mathrm{K}$, dependent on the fibre orientation. This was assumed to be caused by a mean stress applied to the material. In the ongoing research it came out, that the difference of the calibration constants between the two fibre orientations can be ascribed to the used reference measuring system. In [5] strain gauges were used to measure the sum of the principal strains. The composite material PA6 GF30 has a comparatively low stiffness. The stiffness of the strain gauges attached to the specimen is influencing the stiffness of the specimen, leading to an underestimation of the measured strain. The influence of the strain gauges' stiffness on the measured strain depends on the material stiffness and therefore fibre orientation.

\section{Thermoelastic response of PA6 GF30 under fatigue load conditions}

The performance of the material under fatigue load conditions was investigated in a load increase test until failure. Each load step had 1000 load cycles with a loading frequency of $5 \mathrm{~Hz}$. The upper stresses $\sigma_{\mathrm{o}}$ for fibre orientation L started from 7.6 MPa, stepwise increased by $3.06 \mathrm{MPa}$ until failure. For fibre orientation $\mathrm{T} \sigma_{\mathrm{o}}$ was increased from $6.1 \mathrm{MPa}$ in steps by $1.53 \mathrm{MPa}$ until failure, see the upper graphs of Figure 4.
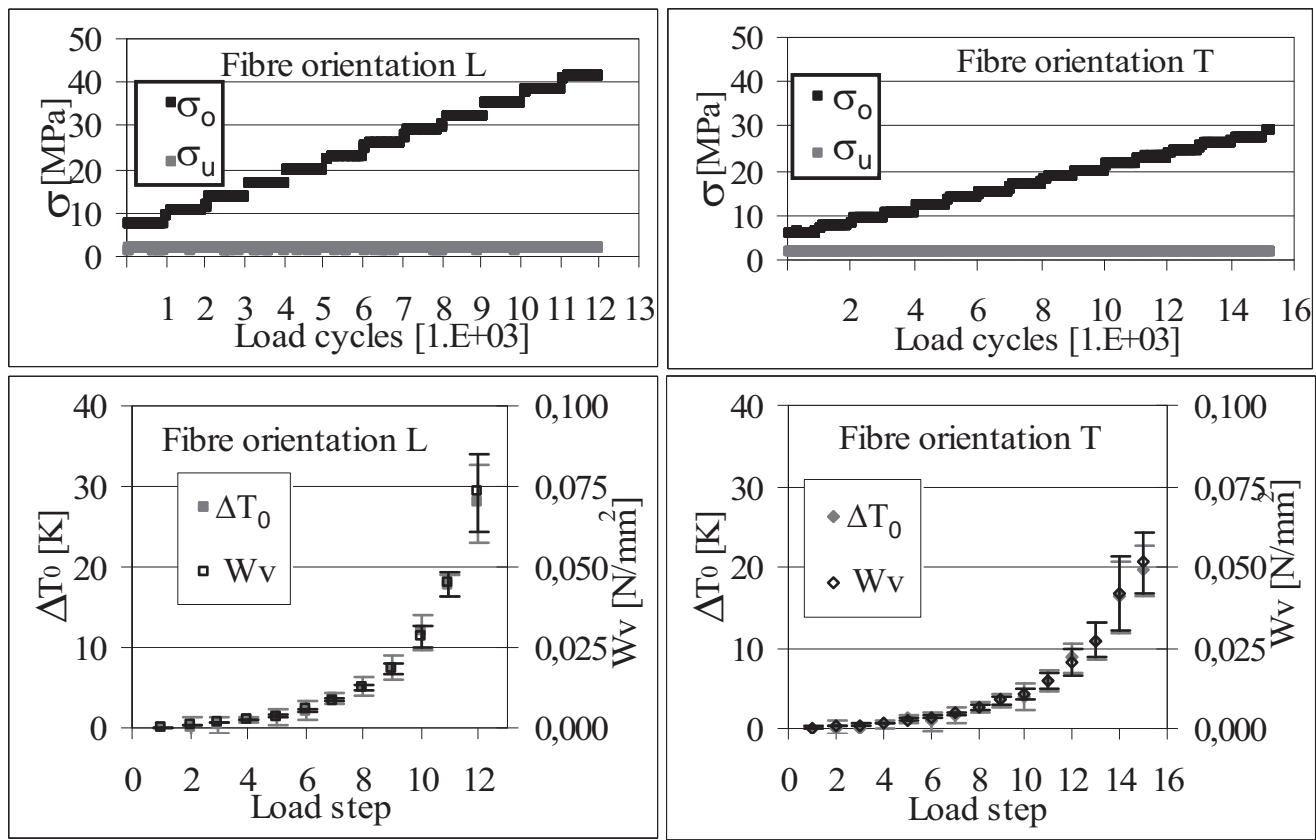

Fig. 4. Load steps of the load increase test until failure, energy loss $W_{v}$ and increase of absolute temperature $\Delta \mathrm{T}_{0}$

The lost mechanical energy during one load cycle is transformed mainly into heat. The mechanical energy loss measured with the LEX is shown together with the increase of the absolute temperature $\Delta \mathrm{T}_{0}$ in the lower graphs of Figure 4 . Notice that $\Delta \mathrm{T}_{0}$ is not the thermoelastic temperature change, but 
the change of the absolute temperature $\mathrm{T}_{0}$. The initial absolute temperature of the specimens was identical to the ambient temperature, $297 \mathrm{~K}$. The temperature was measured with the absolute temperature signal $\mathrm{S}_{\mathrm{DC}}$ of the DeltaTherm TSA-system.

If PA6 GF30 is loaded beyond a specific load level, the hysteresis loop depicted in Figure $2 \mathrm{~b}$ changes significantly. The mechanical energy loss is increasing with increasing load. Still, the energy loss is beyond a specific load level not proportional to the load applied to the material. The increase of the mechanical loss energy and the temperature with increasing load can not be ascribed only to the viscoelastic properties of the material. Especially the disproportional high increase in the upper load steps is rather caused by an additional heating due to internal friction caused by a beginning degradation of the composite material.

The degradation of short fibre reinforced thermoplastics under fatigue load conditions occurs with different failure phenomena, dependent on the fibre-orientation, the fibre aspect ratio, fibrematrix adhesion, etc.. The different failure phenomena have in common that the stiffness of the composite material is decreasing with ongoing material degradation, which can be measured with the LEX. The thermoelastic signal is proportional to the sum of the principal strains, i. e. it is a measure for the material stiffness as well as the strain amplitude.

The measured strain double amplitude $\Delta \varepsilon$ in the load increase test is shown in the upper graphs of Figure 5 with the black symbols. The change of the strain amplitude between a load step and the preceding load step is depicted with the grey symbols.

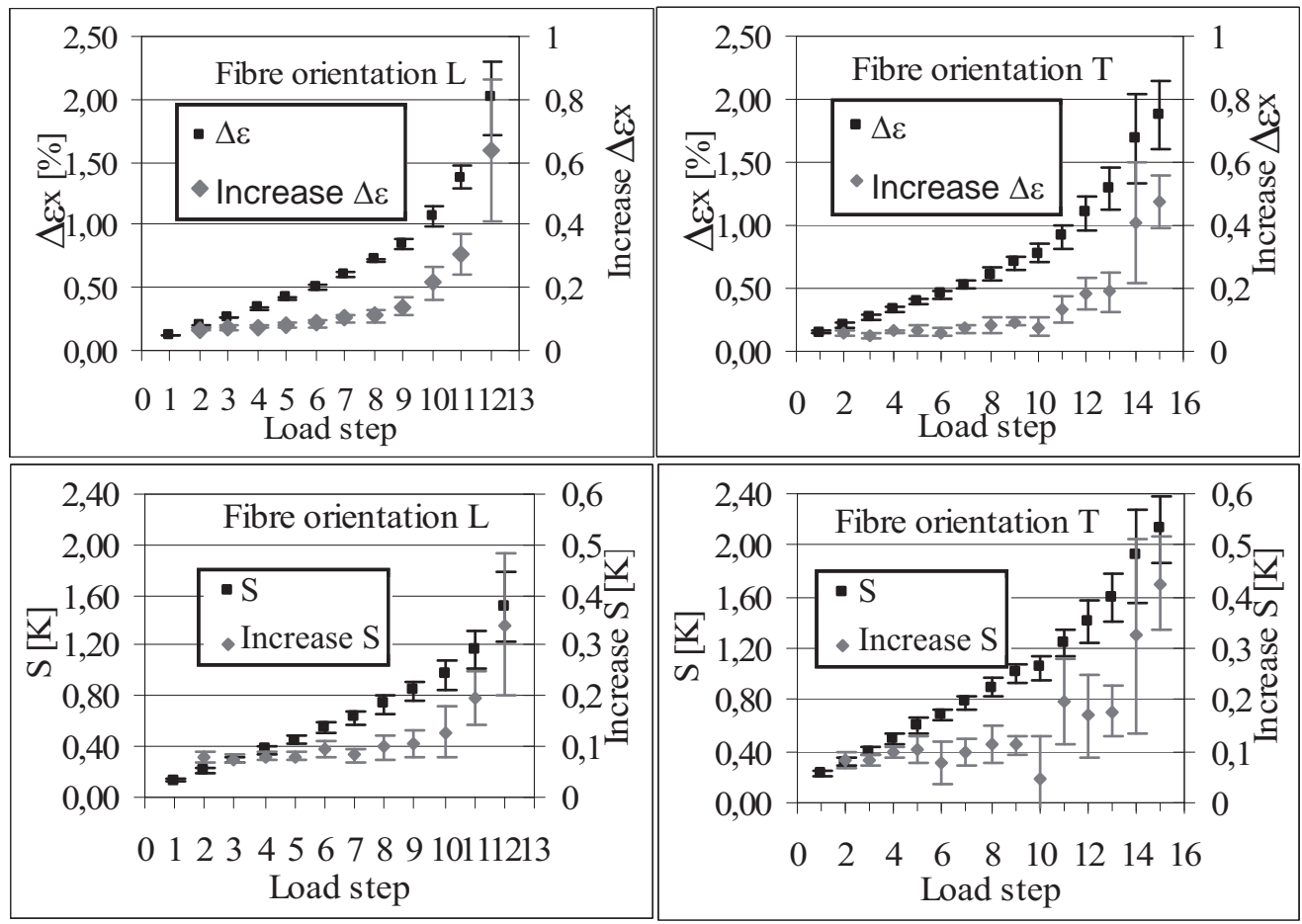

Fig. 5. Strain double amplitude $\Delta \varepsilon$, increase of $\Delta \varepsilon$, thermoelastic signal S and increase of $\mathrm{S}$ for 2 fibre orientations in a load increase test

The strain amplitude is increasing nearly proportional to the load in the lower load steps, from a certain load step is the strain amplitude increasing disproportionally high to the applied load, caused by a decreasing stiffness of the material. The thermoelastic signal S shows the same effect; see the lower graphs of Figure 6. The increase of the signal between two load steps is again depicted with the grey symbols. The load step where the signals are increasing is the critical load step, causing first 
damage in the material. It was identified as the $10^{\text {th }}$ load step $\left(\sigma_{0}=35.2 \mathrm{MPa}\right)$ for fibre orientation $\mathrm{L}$ and the $11^{\text {th }}\left(\sigma_{0}=21.4 \mathrm{MPa}\right)$ load step for fibre orientation $\mathrm{T}$.

Measurements of the acoustic emission (AE) of the material under cyclic load were performed in order to verify the assumption that the measured decrease of the material stiffness is caused by a beginning disruption of the material. The failure of single fibres is resulting in an acoustic event with a specific energy, amplitude and duration. The AE of such an event can be measured by applying an acoustic sensor on the surface of cyclically loaded specimens. A good measure for the occurrence of damage in short fibre reinforced thermoplastics is the total energy of the acoustic event [10]. The results of the AE measurements are shown in Figure 6, the graphs are showing the accumulated energy of all acoustic events measured in the load increase test.
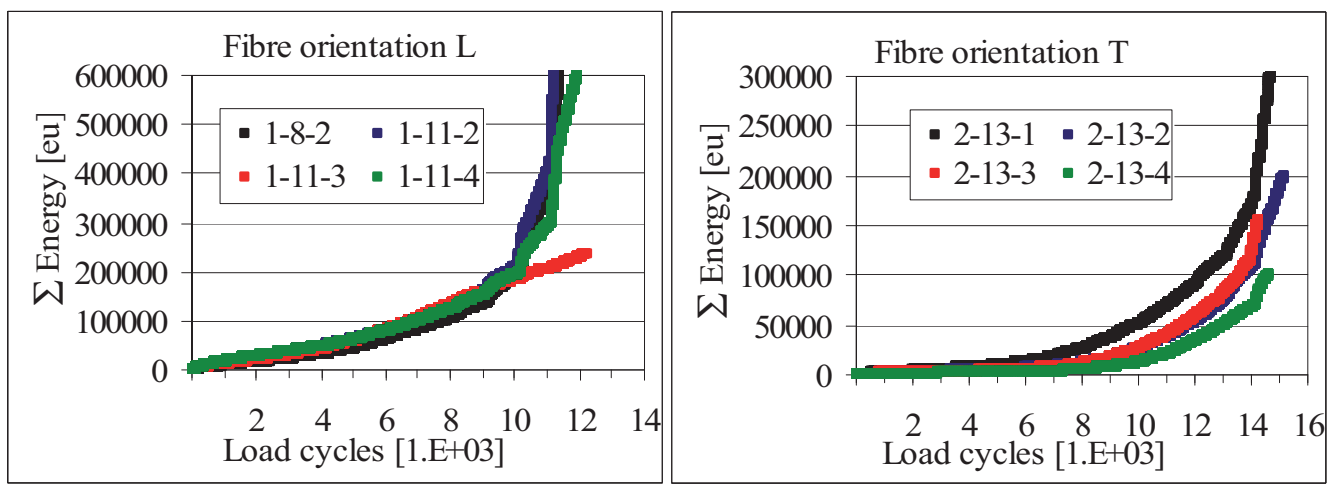

Fig. 6. Accumulated energy of acoustic events of cyclically loaded PA6 GF30 measured with the AE measuring technique in a load increase test

The energy of the acoustic events is increasing from load step 10 for fibre orientation L, the critical load step identified with the TSA. For fibre orientation T a definite load step where the energy of the acoustic events is increasing can not be determined. That is to be explained by the fact that for fibre orientation $\mathrm{T}$ the damage of the material occurs with a different, more ductile failure mode as for fibre orientation L. For fibre orientation L the damage is more likely to be caused by fibre failure, while for fibre orientation T matrix cracks and fibre-matrix debonding may occur.

If the load is higher than the critical load level identified with the TSA, the beginning degradation of the material should result in a lower number of load cycles until failure in a single load fatigue test. To prove this, single load fatigue measurements with loads in the vicinity of the critical load were performed, results are shown in Figure 7.

The number of load cycles until failure of the specimens is displayed on the x-axis. The tests were aborted if no failure of the specimens occurred after 1.0 E6 load cycles for fibre orientation L, resp. 6.6 E5 load cycles for fibre orientation T.

For fibre orientation $\mathrm{L}$ the critical load of the material for cyclic loading was determined exactly with the TSA as $35.2 \mathrm{MPa}$. For an upper stress $\sigma_{0}$ of $32 \mathrm{MPa}$ no failure of the specimens was observed until 1.0 E6 load cycles. The specimens failed after 4.2 E5, resp. 0.8 E5 load cycles if the upper stress was $35.2 \mathrm{MPa}$, the critical load identified with the TSA. For even higher loads the number of load cycles until failure decreased as expected rapidly.

For fibre orientation $\mathrm{T}$ the identification of the critical load for cyclic loading was unfortunately not that exact as for fibre orientation L. The critical load was identified as an upper stress $\sigma_{0}$ of 21.4 $\mathrm{MPa}$, still, the material did not fail at this load until 6.6 E5 load cycles. Nevertheless, if the load was increased to $24.4 \mathrm{MPa}$, the material failed after no more than $0.5 \mathrm{E} 5$ load cycles. 

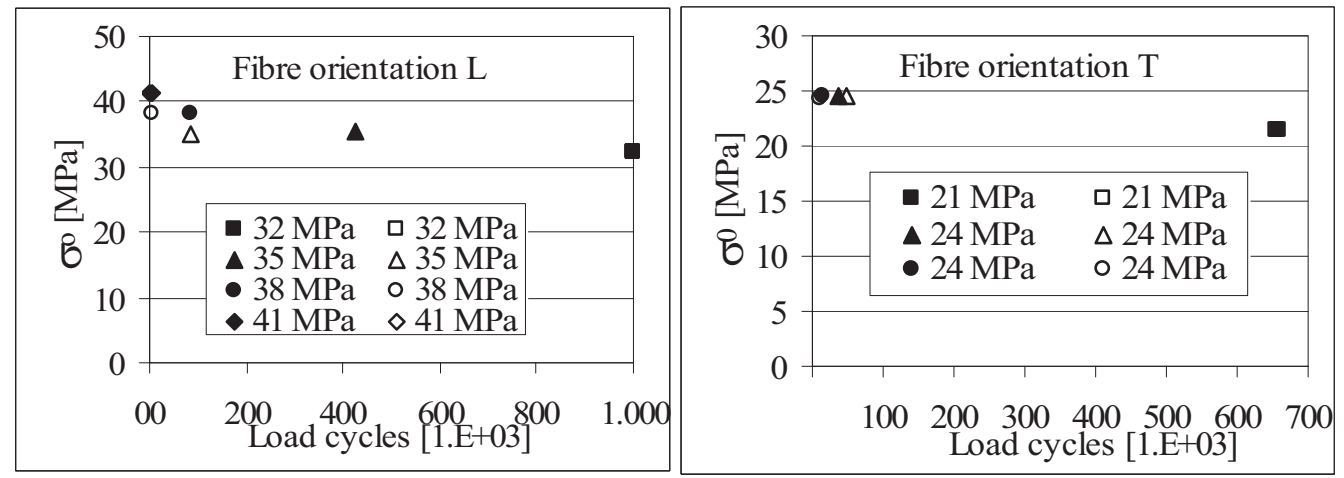

Fig. 7. Load cycles until failure in single step fatigue tests of PA6 GF30

Even though, the identification of the critical load for cyclic loading of short fibre reinforced thermoplastics with the TSA leads to satisfactory results bearing in mind, that the critical load was determined in a load increase test in less than an hour per specimen. A fatigue test in contrast needs a few days for only one load level. Another important advantage of the TSA is that it can be applied to a component of complex shape and strain distribution, as it is a full field measuring technique. It should allow to identify regions on components, where the critical load determined on specimens is reached.

1. Pitarresi, G. und Patterson, E.A.: A review of the general theory of thermoelastic stress analysis, J. Strain Analysis, 38 (5), 405-417, (2003)

2. Dulieu-Barton, J. M, Emery, T. R., Quinn, S. and Cunningham, P.R.: A temperature correction methodology for quantitative thermoelastic stress analysis and damage assessment, Measurement Science and Technology, 17, 1627-1637, (2006)

3. Klavzar, A., Stelzer, G., Renz, R.: Thermal correction of thermoelastic data by a direct calibration of the thermal signal, Measurement Science and Technology, 20 (2), 13 pp, (2009)

4. Hegler, R. P., Altstädt, V., Ehrenstein, G. W.: Einfluß stofflicher Parameter auf die Faserorientierung beim Verarbeiten kurzfaserverstärkter Thermoplaste, Kunststoffe, 76 (9), 766771, (1986)

5. Klavzar A., Jimenez A., Renz R.: Measurement of the Thermoelastic Response of Short-FibreReinforced Polyamide 6 with Application to a Component under Operating Load, J. Strain Analysis, 43 (4), 187-203, (2008)

6. Friebe, H.; Galanulis, K; Erne,O.; Müller, E.: FLC Determination And Forming Analysis by Optical Measurement Systems, FLC-Zurich 06, ETH Zurich, Switzerland, 15.-16. März, 2006

7. Dulieu-Barton, J. M., Eaton-Evans, J., Little, E. G., Brown, I. A.: Thermoelastic Stress Analysis of Vascular Devices, Strain, 44, 102-118, (2008)

8. Emery, T. R., Dulieu-Barton, J. M., Earl, J. S., Cunningham, P.R.: A generalised approach to the calibration of orthotropic materials for thermoelastic stress analysis, Composites Science and Technology, 68, 743-752, (2008)

9. Reese, O.: Ortsaufgelöste Hysteresismessung mit Hilfe eines Laser-Extensometers. Verfahren und Vorrichtung zur Ermittlung der mechanisch-dynamischen Eigenschaften von Werkstücken, Dissertation, Technische Universität Kaiserslautern, (2000)

10. Wolters, J.: Anwendung der Schallemissions-Messtechnik zum Beschreiben von Versagensmechanismen in partikelgefüllten Thermoplasten, Fortschr.-Ber., VDI-Reihe 5, Nr. 163., VDI-Verlag, Düsseldorf, (1989) 